\title{
ENHANCING MEDIASTINAL MASS AT MR: CASTLEMAN DISEASE
}

\author{
Ming Lin Hsieh, ${ }^{* 1}$ Leslie E. Quint, ${ }^{*}$ John M. Faust, ${ }^{* 2}$ and Jennifer E. Turner $\dagger^{3}$ \\ *Department of Radiology, University of Michigan Medical Center, Ann Arbor, MI 48109, USA, and \\ †Department of Radiology, University of Mississippi Medical Center, Jackson, MS 39216, USA
}

\begin{abstract}
We report a case of an enhancing middle mediastinal mass imaged by MR and CT. Repeated nondiagnostic biopsies in this 25-yr-old male patient had resulted in profuse hemorrhage. Final pathological diagnosis after thoracotomy and debulking was Castleman disease. This is a rare, benign lymphoproliferative disorder of uncertain etiology. Histological varieties include the hyaline vascular type $(80-90 \%)$ and the plasma cell type (10-20\%). Cure is generally accomplished via surgical excision. This entity should be included in the differential diagnosis of enhancing mediastinal lesions, prompting awareness of the potential for bleeding complications following biopsy.
\end{abstract}

Keywords: Castleman disease; Hamartoma; Lymphatic system; Magnetic resonance (MR); Computed tomography (CT); Magnetic resonance imaging (MRI).

Castleman discase is a rare, benign lymphoproliferative disorder of uncertain etiology, as evidenced by the multiple synonyms of the disease, including angiofollicular lymph node hyperplasia, lymphoid hamartoma, benign giant lymphoma, and follicular reticuloma. The majority of reported cases are solitary masses in the thorax, predominantly in the hila and mediastinum. ${ }^{1}$ However, the disease can occur in extrathoracic lymph nodes, as well as in non-lymphoid tissues such as the central nervous system. ${ }^{2}$ Cure is generally accomplished via surgical excision. Approximately 80$90 \%$ of cases are of the hyaline vascular histological type, and the remainder are of the plasma cell type. The latter type is often associated with systemic signs and symptoms, including fever, increased sedimentation rate, anemia, hyperglobulinemia, and leukocytosis. ${ }^{1}$ CT findings of several cases of Castleman disease have been previously described, ${ }^{3,4}$ however there is scant information regarding MR imaging characteristics of this disease. ${ }^{5,6}$ We describe one case of mediastinal Castleman disease imaged by $C T$ and contrast enhanced MR.

\section{CASE REPORT}

An otherwise healthy 25 -yr-old man presented with a several year history of recurrent pneumonias and re- cent hemoptysis unresponsive to antibiotic therapy. Chest radiographs revealed a right hilar mass and widening of the carina, suggestive of a subcarinal mass. At bronchoscopy, there was a large submucosal mass at the carina as well as a submucosal and endobronchial, friable mass that nearly occluded the right mainstem bronchus. Bronchoscopic washing, brushing, and biopsy led to copious bleeding; pathology was nondiagnostic. Subsequent contrast enhanced thoracic $C T$ scanning demonstrated a $6 \times 6 \times 10 \mathrm{~cm}$ high attenuation, subcarinal mass which compressed the carina from below and encircled the right lower lobe bronchus (Fig. 1). The mass was fairly homogeneous except for multiple coarse calcifications. There were a few associated nonenlarged precarinal and right paratracheal lymph nodes.

Thoracic MR imaging at $1.5 \mathrm{~T}$ was done using coronal $T_{1}$-weighted and axial $T_{2}$-weighted images before contrast, and axial $T_{1}$-weighted images following intravenous (IV) administration of gadopentetate dimeglumine. Compared to muscle, the mass was slightly hyperintense at $T_{1}$-weighting and extremely hyperintense at $T_{2}$-weighting, and it showed marked enhancement after contrast administration (Fig. 2). Lesionto-fat signal intensity ratios were $\mathbf{0 . 9 3}$ on precontrast
RECEIVED 4/8/92; ACCEPTED 1/12/93.

Address correspondence to Leslie E. Quint, MD, Department of Radiology, Box 0030, University of Michigan Hospitals, 1500 E. Medical Center Drive, Ann Arbor, MI 48109-0030.
Present addresses: ' Department of Radiology, William Beaumont Hospital, Royal Oak, MI; ${ }^{2}$ Department of Radiology, Erlanger Medical Center, Chattanooga, TN; and ${ }^{3}$ Department of Radiology, University of Mississippi Medical Center, Jackson, MS. 


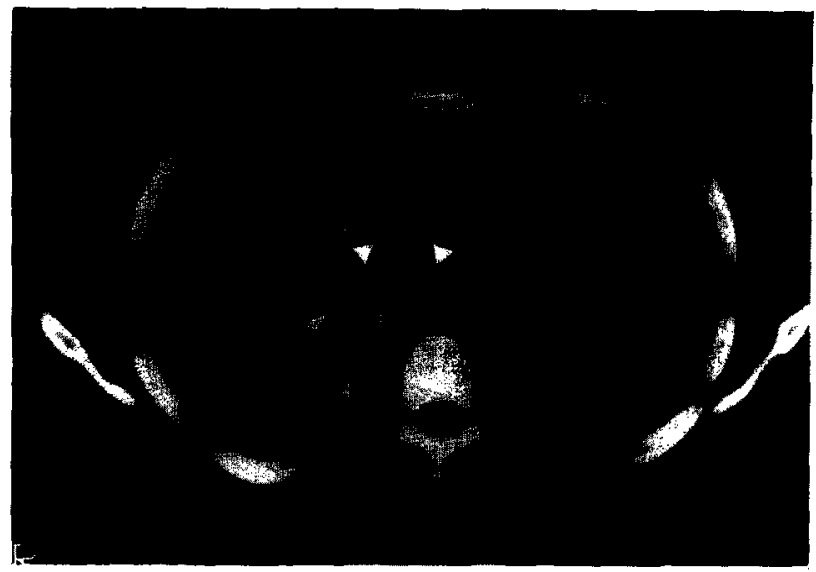

Fig. 1. Contrast enhanced CT shows a high density subcarinal mass (arrowheads) with central calcifications. The mass encases and possibly invades the bronchus intermedius (arrow).

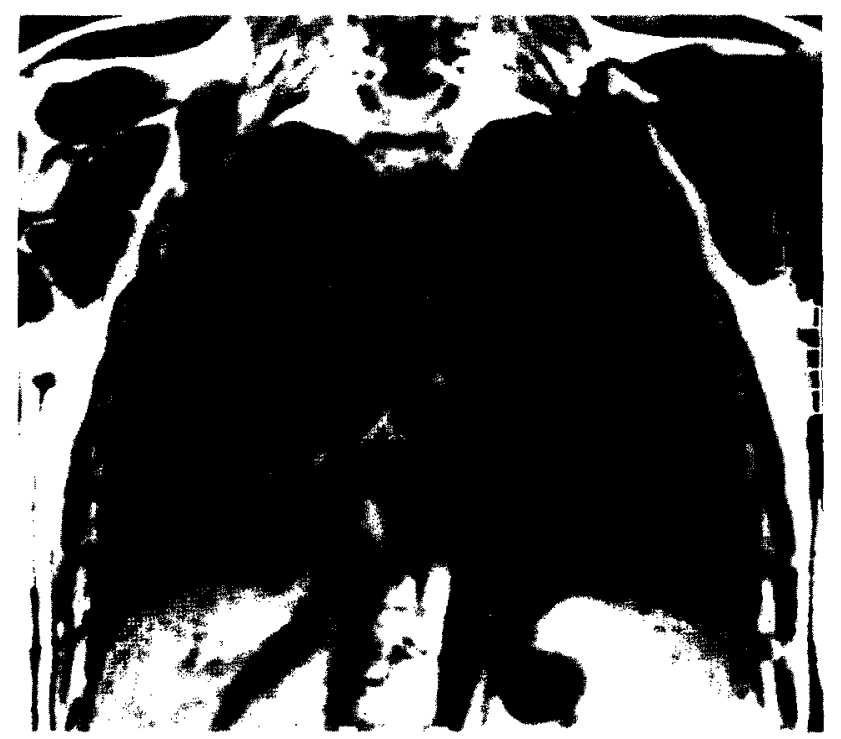

(A)

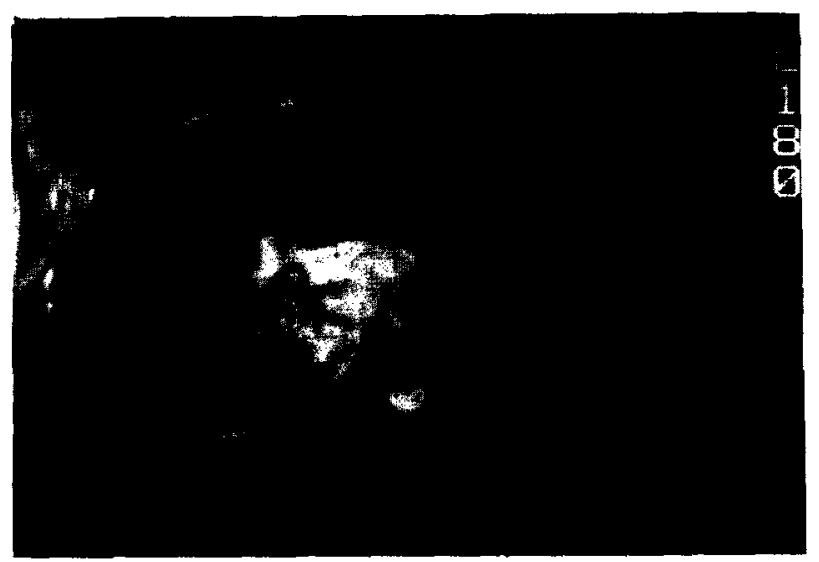

(B)
$T_{1}$-weighted images, 1.05 on $T_{2}$-weighted images, and 0.93 on $T_{1}$-weighted postcontrast images. Intratumoral calcifications seen at CT were difficult to appreciate at MR.

Mediastinoscopy and mediastinotomy resulted in difficult hemostasis; tissue obtained was nondiagnostic. Subsequent thoracotomy revealed a large friable, highly vascular subcarinal mass that extended into the carina and was felt to be unresectable. The mass was debulked and frozen section tissue analysis reported possible Hodgkin disease. The final pathological report, after routine histological and electron microscopic analysis, was plasma cell variant of Castleman disease. Postoperative bone marrow biopsy and abdominal CT scan were negative. Given the unresectable nature of the mass, the patient was treated with radiation therapy ( 3600 centigray) to the mediastinum. This resulted in at least $75 \%$ reduction in tumor mass at MR. Lesion-

Fig. 2. Pre- and postcontrast $M R$ images at $1.5 \mathrm{~T}$. (A) Coronal $T_{1}$-weighted (SE 700/20) image demonstrates widening of the carina and possible occlusion of the bronchus intermedius and proximal right lower lobe bronchus by the large mass (arrows). Central calcifications are not identified. (B) The mass (arrows) is markedly hyperintense on axial $T_{2}$ weighted images (SE 2500/80). (C) Prominent enhancement is seen after administration of gadolinium ( $T_{1}$-weighted; SE $800 / 72$ ).

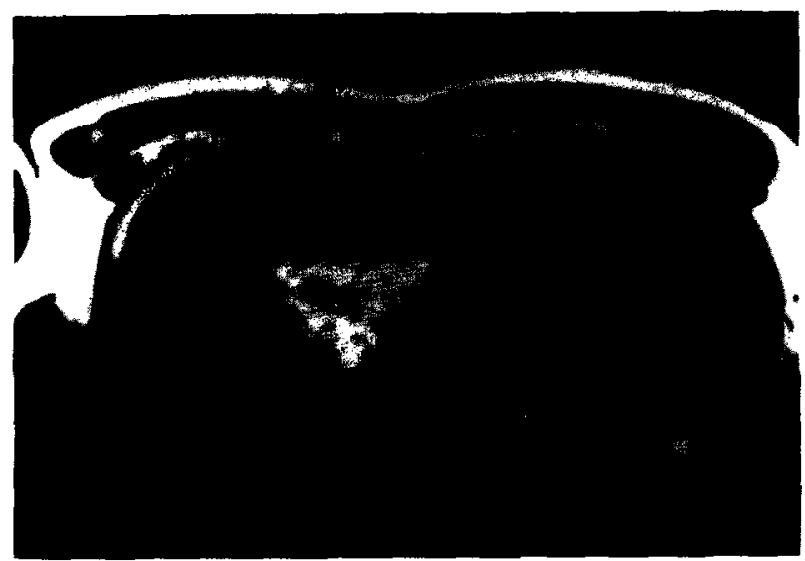

(C) 
to-fat signal intensity ratios after radiation therapy were 0.77 on precontrast $T_{1}$-weighted images and 0.96 on $T_{2}$-weighted images. Contrast enhanced images were not obtained at this time. Thirteen months following surgery, the patient was asymptomatic.

\section{DISCUSSION}

The CT findings in this case agree with the typical findings described in the literature for this disease entity, that is, a well-defined, solitary mass with homogeneous, intense contrast enhancement and scattered, dense calcifications, as well as small adjacent lymph nodes. ${ }^{1,3,4}$

To our knowledge, there are only two prior cases of chest or abdominal Castleman disease that have been imaged with MR and reported in the literature; neither of these cases used IV contrast. ${ }^{5,6}$ De Geer at al. described one patient with mediastinal hyaline vascular type Castleman disease imaged at $0.35 \mathrm{~T}^{5}$ The mass/fat ratio was 0.50 at $T_{1}$-weighting and 0.77 at $T_{2}$-weighting. The mass did not show significant contrast enhancement at CT. A case of retroperitoneal Castleman disease reported by Bartkowski and Ferrigni was isointense with muscle at $T_{1}$-weighting and nearly isointense with fat at $T_{2}$-weighting; calcification and contrast enhancement were present at $\mathrm{CT} .{ }^{6}$ Our case showed similar MR imaging characteristics. In addition, hypervascularity of the tumor was confirmed via marked enhancement after administration of gadolinium.

Advantages of MR over CT in this setting include the capability to perform multiplanar imaging; this may be extremely useful in surgical or radiation therapy treatment planning. Moreover, in patients with contraindications to IV urographic contrast (e.g., renal failure or prior contrast reaction), tumor enhancement can be assessed using contrast enhanced MR. One advantage of CT is the ability to detect intratumoral calcifications; the presence of such calcifications may aid in the differential diagnosis.

The differential diagnosis of an isolated subcarinal mass is long; enhancement of the lesion would be consistent with paraganglioma, carcinoid, aneurysm, lymph node enlargement due to metastasis from hypervascular primary tumors, parathyroid tumors, or ectopic thyroid. The presence of calcifications favors paraganglioma, carcinoid, aneurysm, or ectopic thyroid. Lymphomas do not typically show strong contrast enhancement at $\mathrm{CT},{ }^{7}$ and calcification would be unusual in an untreated lymphoma. The morphology of the lesion, the pattern of calcification, the degree of contrast enhancement and the lack of flow void exclude the diagnosis of aneurysm in our case. Ectopic thyroid would not invade the tracheobronchial tree. The imaging features of this lesion are compatible with mediastinal carcinoid or paraganglioma, and silent endocrine tumors of these types could not have been excluded.

Profuse hemorrhage that occurred during tissue sampling in our case is not uncommon in Castleman disease, and pre-resection embolization has been used in some cases. ${ }^{8}$ It is important to include Castleman disease in the differential diagnosis of enhancing mediastinal lesions, in order to alert the surgeon to the possibility of severe bleeding after biopsy or other instrumentation.

\section{REFERENCES}

1. Keller, A.R.; Hochholzer, L.; Castleman, B. Hyalinevascular and plasma-cell types of giant lymph node hyperplasia of the mediastinum and other locations. Cancer $29: 670-683 ; 1972$.

2. Gianaris, P.G.; Leestma, J.E.; Cerullo, L.J.; Butler, A. Castleman's disease manifesting in the central nervous system: Case report with immunological studies. Neurosurgery 24(4):608-613; 1989.

3. Ferreiros, J.; Leon, N.G.; Mata, M.I.; Casanova, R.; Pedrosa, C.S.; Cuevas, A. Computed tomography in abdominal Castleman's disease. J. Comput. Assist. Tomogr. 13(3):433-436; 1989.

4. Fiore, D.; Biondetti, P.R.; Calabro, F.; Rea, F. CT demonstration of bilateral Castleman tumors in the mediastinum. J. Comput. Assist. Tomogr. 7(4):719-720; 1983.

5. de Geer, G.; Webb, W.R.; Sollitto, R.; Golden, J. MR characteristics of benign lymph node enlargement in sarcoidosis and Castleman's disease. Eur. J. Radiol. 6:145148; 1986.

6. Bartkowski, D.P.; Ferrigni, R.G. Castleman's disease: An unusual retroperitoneal mass. J. Urol. 139:118-120; 1988.

7. Magnusson, A.; Andersson, T.; Larsson, B.; Hagberg, H.; Sundström, C. Contrast enhancement of pathologic lymph nodes demonstrated by computed tomography. Acta Radiol. 30(3):307-310; 1989.

8. Walter, J.F.; Rottenberg, R.W.; Cannon, W.B.; Sheridan, L.A.; Pizzimenti, J.; Orr, J.T. Giant mediastinal lymph node hyperplasia (Castleman's disease): Angiographic and clinical features. Am. J. Roentgenol. 130:447-450; 1978. 